Hi gh- speed tangent i al I y vi ewing sof $t$ x-ray camer a to st udy magnet ohydr odynami $c$ fl uct uat i ons i $n$ tor oi dal I y conf i ned pl asmas ( i nvi ted)

\begin{tabular}{|l|l|}
\hline $\begin{array}{l}\text { j our nal or } \\
\text { publ } \mathrm{i} \text { cat } \mathrm{i} \text { on } \mathrm{titl} \text { e }\end{array}$ & Revi ew of Sci ent i f i c I nst r ument s \\
\hline vol une & Vol . 74 \\
\hline number & I ssue 3 \\
\hline page range & pp. 2136- 2143 \\
\hline year & 2003 03 - 01 \\
\hline URL & ht t p: //hdl . handl e. net /10655/8976 \\
\hline
\end{tabular}




\section{AIP Scientific Instruments}

\section{High-speed tangentially viewing soft x-ray camera to study magnetohydrodynamic fluctuations in toroidally confined plasmas (invited)}

S. Ohdachi, K. Toi, G. Fuchs, S. von Goeler, and S. Yamamoto

Citation: Rev. Sci. Instrum. 74, 2136 (2003); doi: 10.1063/1.1537449

View online: http://dx.doi.org/10.1063/1.1537449

View Table of Contents: http://rsi.aip.org/resource/1/RSINAK/v74/i3

Published by the American Institute of Physics.

\section{Related Articles}

Diagnostics of underwater electrical wire explosion through a time- and space-resolved hard $\mathrm{x}$-ray source Rev. Sci. Instrum. 83, 103505 (2012)

A novel technique for single-shot energy-resolved 2D x-ray imaging of plasmas relevant for the inertial confinement fusion

Rev. Sci. Instrum. 83, 103504 (2012)

Near-coincident K-line and K-edge energies as ionization diagnostics for some high atomic number plasmas Phys. Plasmas 19, 102705 (2012)

X-ray backlight measurement of preformed plasma by kJ-class petawatt LFEX laser

J. Appl. Phys. 112, 063301 (2012)

Time-resolved soft x-ray spectra from laser-produced Cu plasma

Rev. Sci. Instrum. 83, 10E138 (2012)

\section{Additional information on Rev. Sci. Instrum.}

Journal Homepage: http://rsi.aip.org

Journal Information: http://rsi.aip.org/about/about_the_journal

Top downloads: http://rsi.aip.org/features/most_downloaded

Information for Authors: http://rsi.aip.org/authors

\section{ADVERTISEMENT}

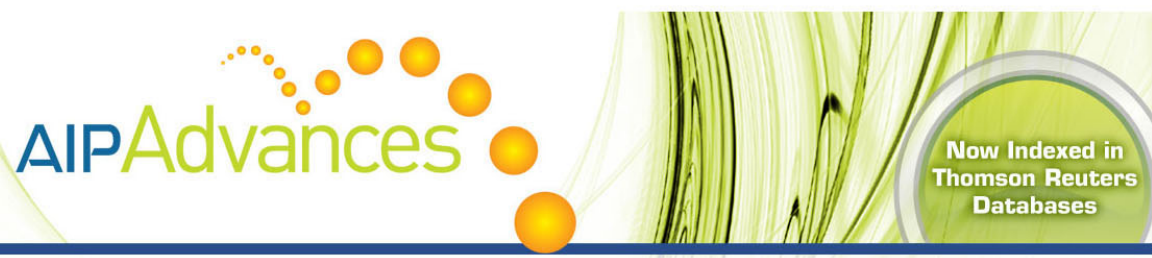

\section{Explore AIP's open access journal: Rapid publication \\ - Article-level metrics \\ Post-publication rating and commenting}




\title{
High-speed tangentially viewing soft x-ray camera to study magnetohydrodynamic fluctuations in toroidally confined plasmas (invited)
}

\author{
S. Ohdachi, ${ }^{a}$ K. Toi, and LHD Experimental Group \\ National Institute for Fusion Science, Toki 509-5292, Japan \\ G. Fuchs \\ Institut für Plasmaphysik, Forschungszentrum Jülich GmbH, EURATOM Association, \\ D-52425 Jülich, Germany \\ S. von Goeler \\ Princeton Plasma Physics Laboratory, Box 451, Princeton, New Jersey 08543
}

S. Yamamoto

Department of Energy Engineering and Science, Nagoya University, Nagoya 464-8603, Japan

(Presented on 11 July 2002)

\begin{abstract}
A high-speed tangentially viewing soft $\mathrm{x}$-ray camera system has been developed and installed on the large helical device (LHD) using a video camera with a maximum framing rate of $13.5 \mathrm{kHz}$. Low-frequency activities, for example, structures with toroidal/poloidal mode number $n / m=1 / 2$, are directly detected with this system. (c) 2003 American Institute of Physics.
\end{abstract}

[DOI: $10.1063 / 1.1537449]$

\section{INTRODUCTION}

Fluctuation patterns inside fusion plasmas are rather complicated. Soft x-ray (SX) tomographic reconstruction methods allow one to deduce the spatial structure of these fluctuations. Although the methods have successfully revealed important fluctuation features, their limitations are also obvious now. (1) Due to the limited number of beams, the diagnostics are insensitive to small scale fluctuations, and furthermore, the integration along the lines of sight acts as a low wave-number filter. (2) In addition, a large number of equally spaced detectors surrounding the plasma is needed to obtain a reliable reconstruction. Due to limited access and scarcity of ports, this is not always possible. An installation inside the vacuum chamber is almost impossible in larger devices, because heat loads onto the detectors are not acceptable, especially for long-duration discharges.

A tangentially viewing camera represents an interesting method to study fluctuations in toroidally confined plasmas and to investigate the typical character of plasma fluctuations. Since most fluctuation patterns vary mainly perpendicular to the magnetic lines of force, disturbances due to the fluctuations tend to have a long wavelength parallel to the magnetic field. If the viewing angle is carefully adjusted, the lines of sight will be almost parallel to the magnetic field-at least in some area-and we can monitor directly the twodimensional (2D) structure of the fluctuations. ${ }^{1}$ Complicated interpretations, which may introduce inevitable errors in the reconstruction process, are not necessarily needed. The advantages of the tangential view hold for higher-number mode fluctuations as well; numerical simulations have shown that for tangential viewing, fluctuations with double-digit mode numbers could easily be resolved. ${ }^{1}$

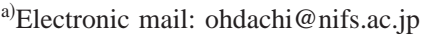

An advantage of a two-dimensional visualization is that we can make full use of established pattern recognition methods to understand complicated phenomena like magnetohydrodynamic (MHD) instabilities. Photography-based measurements of visible light were often employed and have proven to be useful in MHD studies near the plasma edge. ${ }^{2}$ However, in order to study the core plasma, where the temperatures are in the kilo-electron-volt range, we need to observe the x-ray emission. The construction of a fast SX camera was not easy, it took the development of the two key components. (1) A 2D x-ray image convertor with a large sensitive area, developed mainly for medical purposes, provides an output of fast optical images essential for fluctuation studies. (2) The other component is a fast-framing video camera, which is realized by a parallel readout from a complementary metal-oxide semiconductor sensor. These have enabled us to develop a camera which can catch soft $\mathrm{x}$-ray fluctuations in the kilohertz range.

Since 1997, we have developed a camera system in collaboration with the Princeton Plasma Physics Laboratory (PPPL) and the Institut für Plasma Physik at the Forschungszentrum Jülich. ${ }^{3}$ Experimental results obtained on TEXTOR-94 tokamak with a slower framing video camera have been reported elsewhere. ${ }^{4}$ Here, we first report experimental results with a high-speed camera obtained on the large helical device (LHD) in the National Institute for Fusion Science.

The LHD is an $1=2$ Heliotron-type device with a pitch number $m=10$. It is a superconducting machine with major radius $R$ of 3.5 to $3.9 \mathrm{~m}$, and an averaged minor radius $a$ $=0.6 \mathrm{~m}$. The rotational transform $t$ is an increasing function of the averaged minor radius $\rho$; in standard configurations it extends from about 0.4 at the center to about 1.4 at the edge. MHD instabilities located at the rational surfaces $t=1 / 2(\rho$ $\sim 0.5)$ and 1/1 $(\rho \sim 0.9)$ are of importance, especially for 


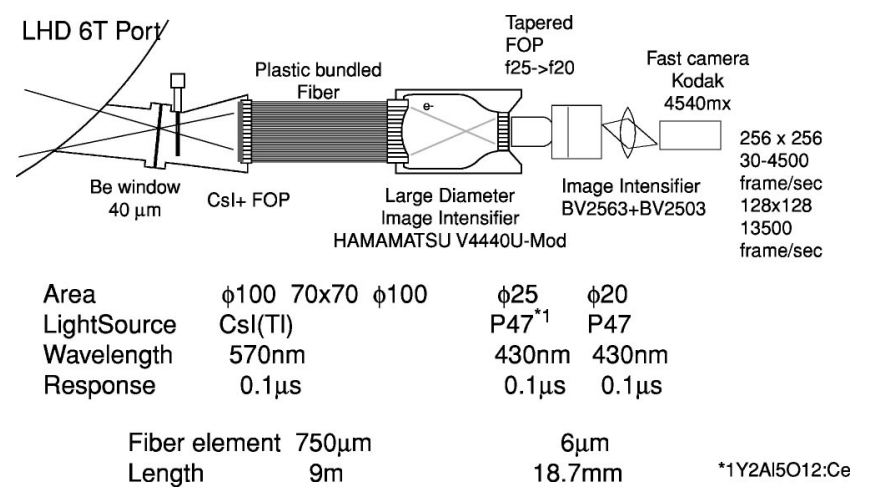

FIG. 1. Basic arrangement of the tangentially viewing SX camera.

inward-shifted plasmas ( $R_{\text {axis }}$ of 3.5 to $3.6 \mathrm{~m}$ ) which have unfavorable MHD properties. ${ }^{5}$ Detailed descriptions will be given for the camera in Sec. II and the data analysis in Sec. III. The scheme to extract fluctuating components from the video data is discussed as well.

\section{EXPERIMENTAL SETUP}

\section{A. Camera system}

The basic concept of the camera is presented in Fig. 1. It consist of a pinhole with a 1-mm-diam camera and a $10-\mathrm{cm}-$ diam scintillator screen made up by a fiberoptic plate coated with CsI $(50-\mu \mathrm{m}$ in thickness). The scintillator screen converts the soft $\mathrm{x}$-ray image into a visible one. A 9-m-long optical fiber bundle [Toray PGR-FB750 $(0.75-\mu \mathrm{m} 100$ $\times 100$ fibers) $]$ is used to guide the light away from the region with a strong magnetic stray field. The light is first amplified using an electron-beam-imaging amplifier (Hamamatsu: $4440 \mathrm{U}-\mathrm{mod}$ ), thereby reducing the image size to $2.5 \mathrm{~cm}$ in diameter. The image is further reduced by a tapered fiberoptic plate and subsequently intensified by a multichannel plate (MCP: Proxitronic: BV2563) followed by a diode (Proxitronic BV2503). Finally, the image is recorded by a highspeed video camera (Kodak: ECTAPRO 4540mx). The framing rate of the camera ranges from $30 \mathrm{~Hz}$ to $4.5 \mathrm{kHz}$ for full resolution $(256 \times 256$ pixels $)$, and extends to $13.5 \mathrm{kHz}$ for a reduced frame of $128 \times 128$ pixels.

We have modified our original plan ${ }^{6}$ by the following actions. (1) The interface to the LHD device has been changed to reduce the visible stray light. The scintillator screen is attached to a separated vacuum chamber, which looks into the LHD main chamber through a vacuum-tight beryllium window ( $25 \mu \mathrm{m}$ thick). Beryllium foils of up to a thickness of $40 \mu \mathrm{m}$ are used if additional filtering is desired. (2) The second image intensifier has been replaced by a twostage amplifier (BV2563 + BV2503), whereby the input window is directly coupled to its tapered fiberoptic plate. Because of the higher transfer efficiency due to the direct coupling, the S/N ratio has significantly improved. The total time response of the system is improved as well; the image intensifiers now use P-47 phosphors (instead of P-20) that have a response time of the order of microseconds.

\section{B. Control system}

The camera system is controlled by an UNIX-based (FreeBSD 3.3) PC (Fig. 2). Since this PC is located in the

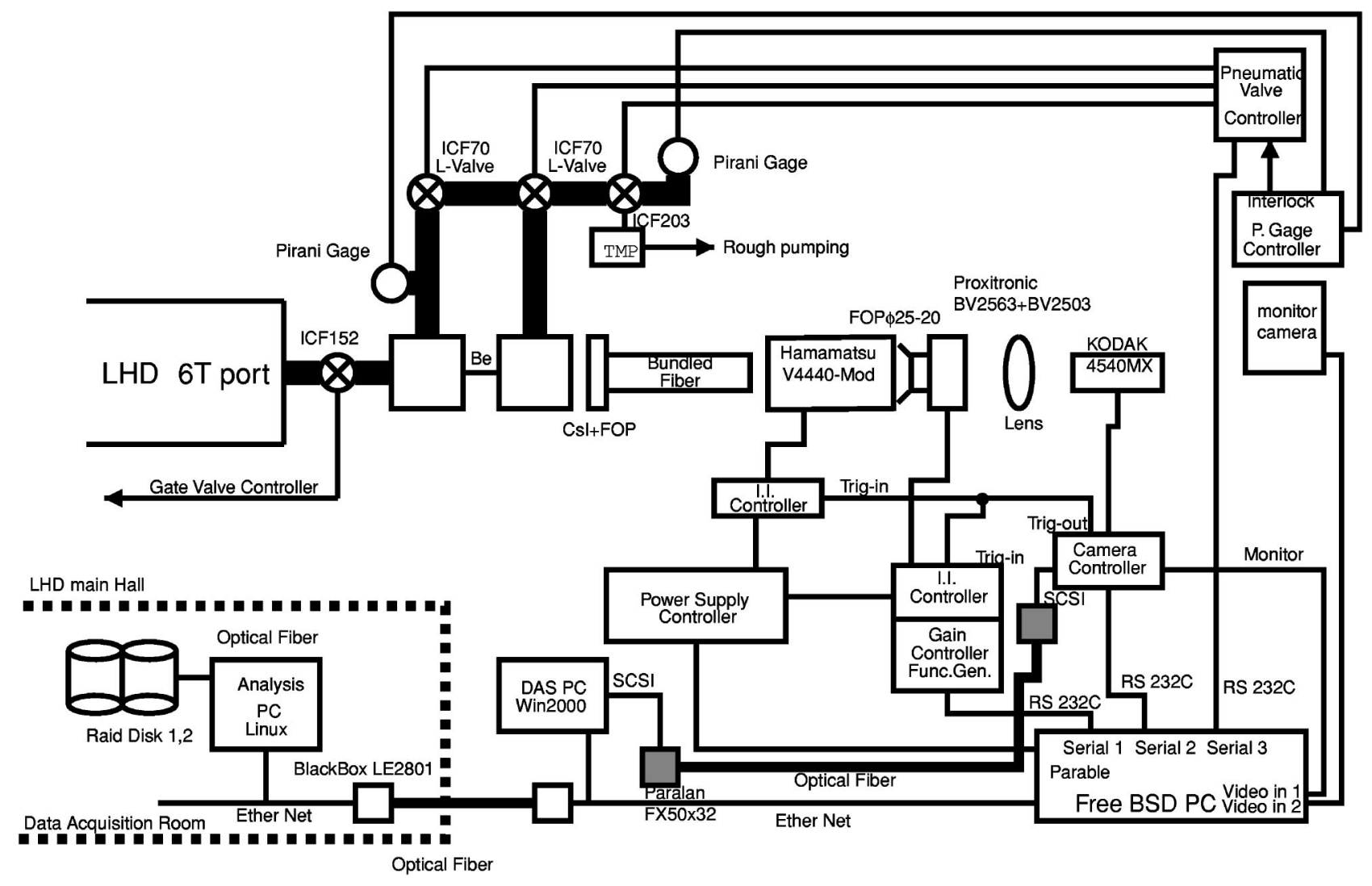

FIG. 2. Control diagram for the camera system. 

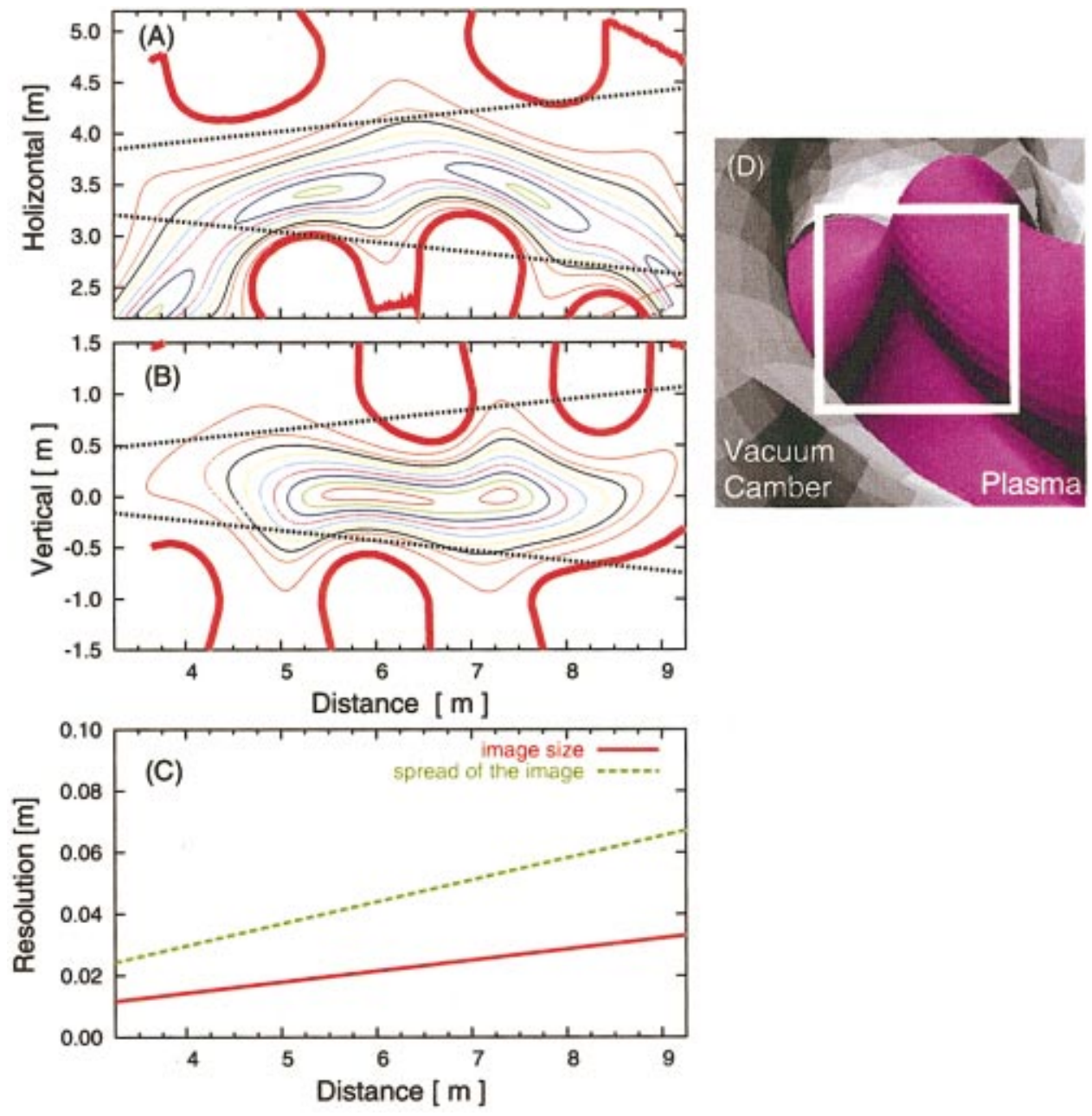

FIG. 3. (Color) The lines of sight together with flux surfaces of the LHD plasma are shown in (A) horizontal cross section and (B) vertical cross section. Thick lines in (A) and (B) indicate the inner walls of the plasma. Size of the plasma elements which are covered by a one pixel in the detectors are shown in (C). Its extent, due to the finite size of the pinhole, is also shown in order to give information about the resolution within the plasma. View from the tangential port is shown in (D). The measuring area is indicated by a white frame.

experimental hall, to avoid damages from the stray magnetic field its storage is replaced by a solid-state disk (256 MB). The PC controls the following systems: (1) the power supplies of the whole system, (2) the framing rate and the frame

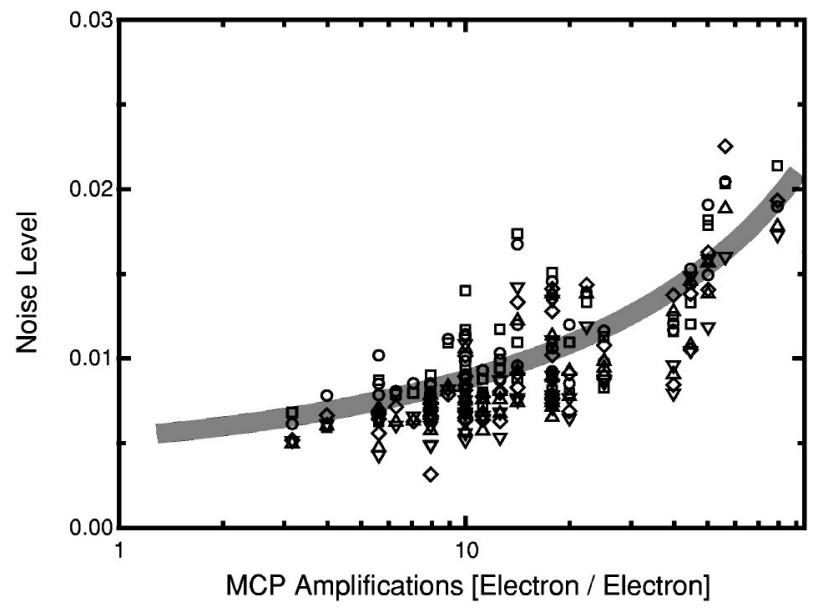

FIG. 4. Noise level as a function of the amplification factor at the MCP plate. size of the video camera, (3) the gains of the image intensifiers [applied voltage to the MCP], and (4) the valves of the vacuum pumping system. In order to use the full dynamic range ( 8 bit) of the fast camera, we need to carefully adjust the framing rate of the camera as well as the gain. An NTSC

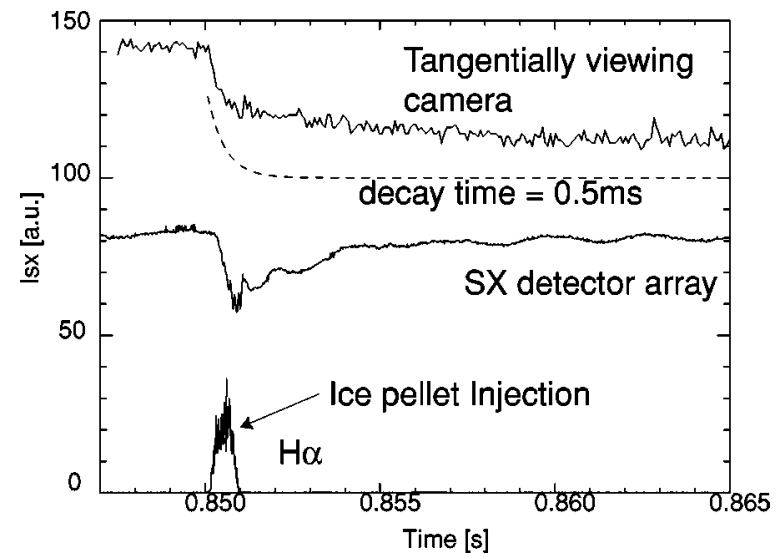

FIG. 5. Time evolution of the camera data. Data from the PIN-diode detector array and $H_{\alpha}$ signal are also shown for comparison. 

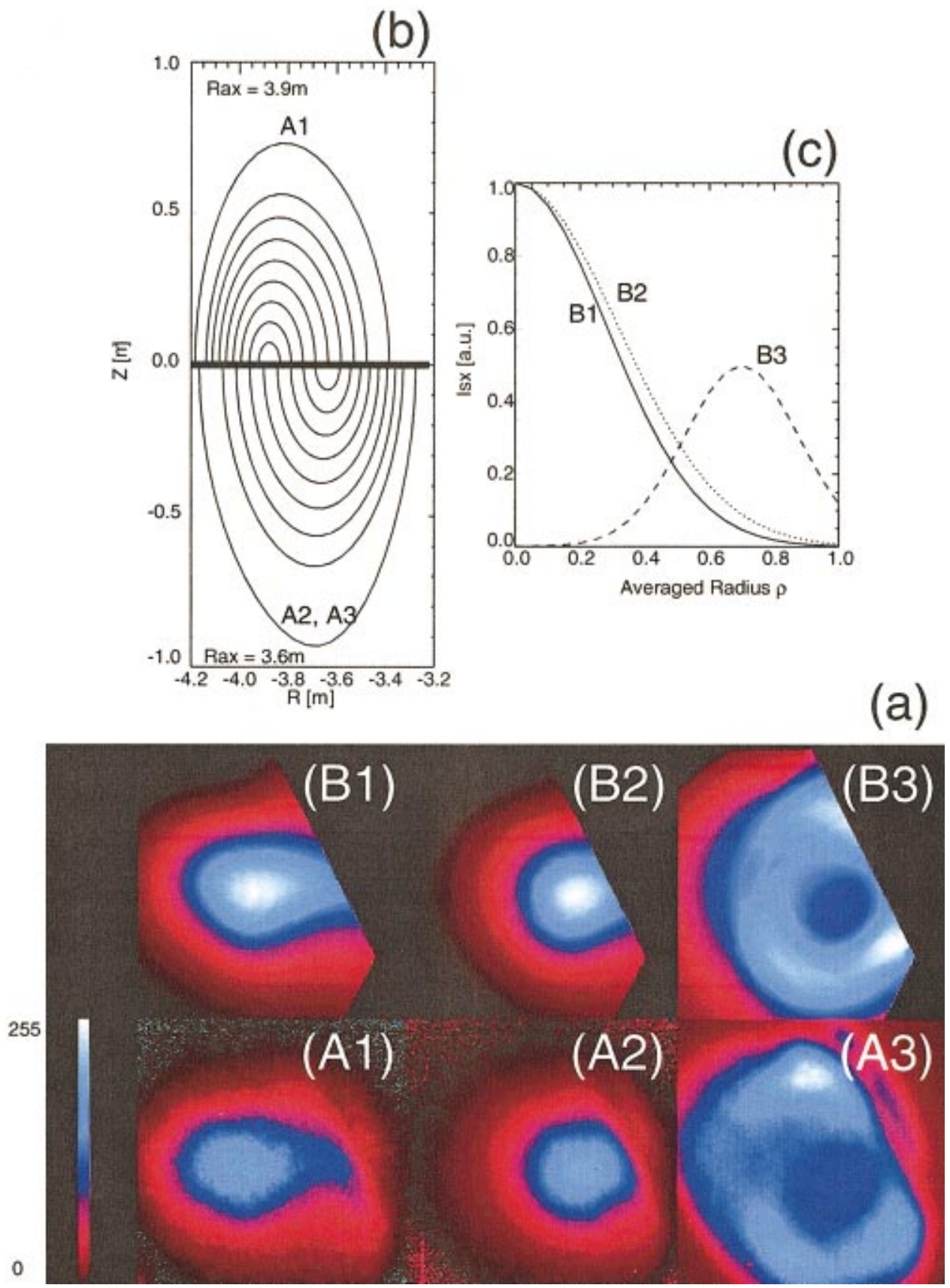

FIG. 6. (Color) Sample images taken by this camera are shown in (A1)-(A3) of (a), and simulated images assuming flux surfaces are shown in (B1)-(B3) of (a). In the simulations, the shape of the flux surface, whose shape at a vertically elongated section is shown in (b), and the radiation profile (c), are used.

monitor signal from the camera-controller is captured by the PC to provide a quick check of the images in the control room; in this way we can quickly infer the gain and the framing rate for the next discharge.

The camera-controller has an internal memory of 512 MB to store the video images; it keeps 8192 frames for the full framing mode ( $256 \times 256$ pixels) and 32768 frames for the half frame mode $(128 \times 128$ pixels $)$. Therefore, we can record video data about 1.8 and 2.4 seconds with framing rates of 4.5 and $13.5 \mathrm{kHz}$, respectively. Data are acquired via a SCSI connection on optical fibers by a Windows-2000 PC (DAS PC in Fig. 2) following each discharge. The stored images are backed up to RAID-5 disk arrays ( $\sim 450 \mathrm{~GB})$ which are controlled by a Linux-based PC (Analysis PC in
Fig. 2) every week. The narrow bandwidth of the optical SCSI connection is the bottleneck of the system. The rate is about $500 \mathrm{kB} / \mathrm{s}$. Usually, to minimize the transfer time, only the interesting part from the complete data set is stored.

\section{Characteristics of the camera}

The camera is installed on a tangential port $(6 \mathrm{~T})$ port of LHD. The lines of sight are shown in Fig. 3. In order to estimate the noise level, the number of the photons are examined, assuming that the radiation is caused only by the bremsstrahlung. In this configuration, $7 \times 10^{8} \mathrm{~s}^{-1}$ photons from the plasma are absorbed by a $(0.4 \mathrm{~mm})^{2}$ area of the scintillator; that part is a $1 / 256 \times 1 / 256$ of the screen con- 


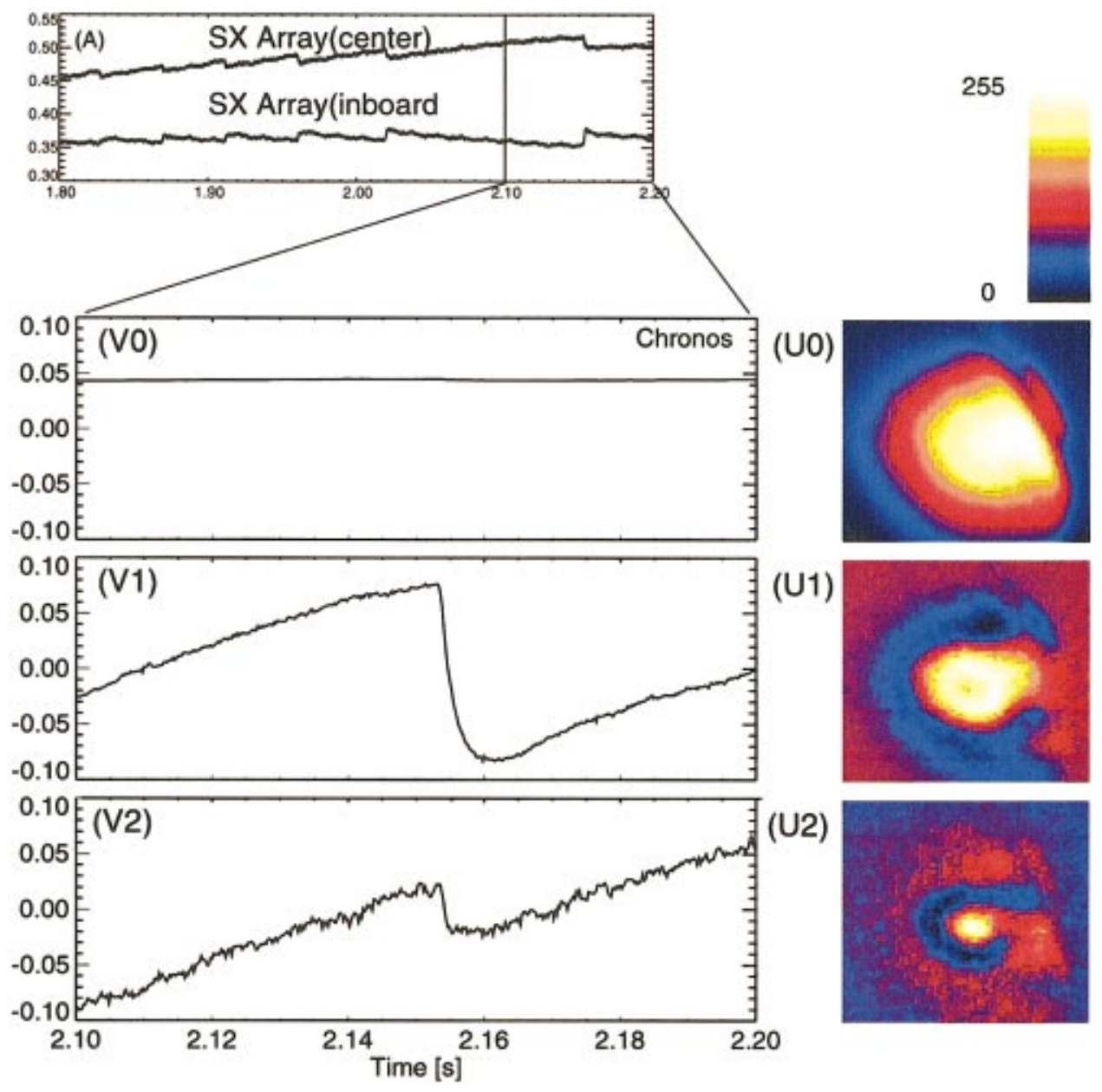

FIG. 7. (Color) Examples of SVD components at a sawtooth-like crash measured by SX detector arrays are shown in (A). Expanded views of chronos (V0-V2) and corresponding topos (U0-U2) are also shown.

nected through optical fibers to one pixel of the video camera. The averaged electron temperature $\left(\bar{T}_{c}\right)=2.0 \mathrm{keV}$, the averaged electron density $\left(\bar{n}_{c}\right)=2.0 \times 10^{19} \mathrm{~m}^{-3}$, and the effective charge $Z_{\text {eff }}=2$ are assumed. Only $4 \%$ of the photons will reach the entrance window of the first image intensifier, whereas the others are lost in between. $1.6 \times 10^{5}$ photons per frame $\left(N_{p}\right)$ are utilized in the measurements, with a $4.5 \mathrm{kHz}$ framing rate. The noise level is estimated as $\Delta N_{p} / N_{p}$ $=\sqrt{N_{p}} / N_{p} \sim 1 \%$. In this pessimistic estimate, only the bremsstrahlung was taken into account; these noise levels are at the lower limit that allows us to study fluctuations. The noise level was measured as a function of the amplification factor of the MCP (Fig. 4). It is about $1 \%$ for a moderate amplification and not quite as large as the estimate. Fluctuations with a relative amplitude exceeding $2 \%$ can be detected by our system.

The resolution of the system is actually not determined by the pixels on the video camera, but by those on the plastic fiber bundle $(100 \times 100)$. For this reason, in order to analyze the fluctuations, we use "super pixels," that is, an average over $4 \times 4$ pixels. This reduces the noise level by a factor of 4 .

The time response of the camera system has been checked in a pellet injection experiment, where the plasma parameters change rapidly. The time evolution of the intensity of the SX radiation as measured by the camera (framing rate $13.5 \mathrm{kHz}$ ) is shown in Fig. 5. A cooling of the plasma just after the injection is clearly observed. The decay time constant is less than $0.5 \mathrm{~ms}$; this agrees with the measurement from the PIN-diode arrays, ${ }^{7}$ where the maximum frequency is about $300 \mathrm{kHz}$. The total response of the camera turns out to be adequate to study fluctuations in the range of several kilohertz. The upper limit is due to the framing rate of the video camera.

Examples of images with different plasma position are shown in Fig. 6(a). The magnetic axis $R_{a x}$ is located at $3.9 \mathrm{~m}$ (A1) and $R_{a x}=3.6 \mathrm{~m}$ in (A2) and (A3). The images lack the right-top and the right-bottom corners because the plasma is obscured by some parts within the tangential port. (B1)-(B3) give simulated images, whereby we assumed flux surfaces, as in Fig. 6(b), together with radiation profiles. ${ }^{6}$ The hollow radiation profile (A3) is caused by strong neon puffing. The simulated images agree well with the measured data although the simulated images have only a resolution of 64 $\times 64$. This is consistent with the resolution of the imaging fiber $(100 \times 100)$. 


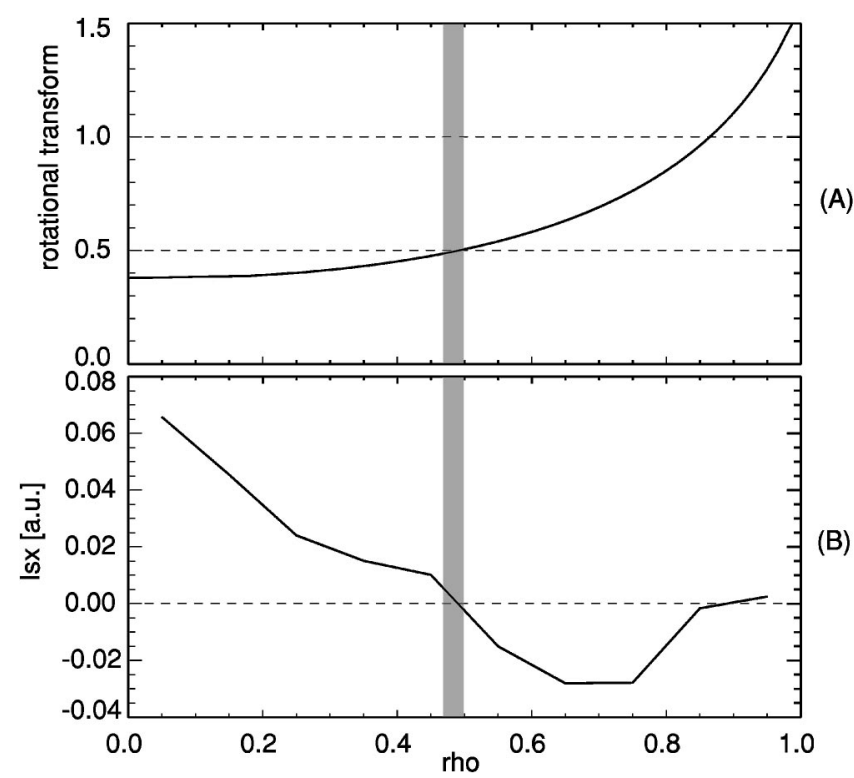

FIG. 8. The rotational transform profile of the plasma with the sawtoothlike phenomena are shown in (A). The radiation profile which is reconstructed from Fig. 7 (U1) are shown in (B).

\section{DATA ANALYSIS}

The extraction of the fluctuating components from video signal is an important issue for fusion devices. In our case, the coherent fluctuation component is comparable to or even lower than the noise level. Nevertheless, the method of singular value decomposition (SVD) allows one to identify coherent fluctuations in space and time within a background of noise. ${ }^{8,9}$ By SVD, a matrix $A(M \times N)$ made up of $N$ time series of $M$ frames is decomposed into three matrices, $U(M \times N), V(N \times N)$ and a diagonal matrix $W(N \times N) ; A$ $=U W V^{t}$. The columns of $U$ and $V$ are spatial and temporal eigenvectors and are called topos and chronos, respectively. A time series $\boldsymbol{a}_{i}$ can be written by a combination of independent components of the topos and the chronos, as

$$
\boldsymbol{a}_{i}=w_{1} \times v_{i 1} \times \boldsymbol{u}_{1}+w_{2} \times v_{i 2} \times \boldsymbol{u}_{2}+\cdots+w_{m} \times v_{i m} \times \boldsymbol{u}_{m} .
$$

As SVD and the Radon transform ${ }^{10}$ are both linear operations, one can reconstruct the local pictures from the topos only, ${ }^{9}$ which are a very small number as compared to the many frames we took for each discharge. The SVD method was first used for the purpose of analyzing signals from the magnetic probes and the SX detector array. ${ }^{8}$ It was furthermore successfully applied to pick up fluctuating components within $2+1$ dimensional data, for example, tomographic images in W7-AS stellarator ${ }^{11}$ and the beam emission spectroscopy in D-IIID tokamak. ${ }^{12}$ This does apply as well to the images taken by the tangential camera; it gives a spatial structure, topos, and its time evolution simultaneously. When the fluctuations in LHD plasmas are caught well by our camera, the topos and their evolution tend to become compressed into smaller data sets. Two examples will be presented.

The camera data have been processed as follows. Before the SV decomposition, the dark current components are removed and averaged over $4 \times 4$ pixels. The averaging not only reduces the noise, but also improves the dynamic range of the camera. Therefore, images are treated as $64 \times 64$ rather than $256 \times 256$ pixel frames. After removing the unnecessary part, for example, top-right corner of the image, SV decomposition is performed.

Three SVD components at the crash of the sawtooth-like phenomena ${ }^{13}$ are shown in Fig. 7. The first and largest component, V0 and U0, represents the global time evolution of the plasma. From the second topo (U1), the decrease in the core region and increase in the edge region-just like a reversed Mexican hat wavelet-is observed during relaxation. In LHD, sawtooth-like relaxations often take places on the $t=1 / 2(\rho \sim 0.5)$ rational surface. The reconstructed radial profile from $\mathrm{U} 1$ is shown in Fig. 8(B). The inversion radius agrees well with the $t=1 / 2$ location [Fig. 8(A)]. The third component (U2) has a peculiar shape. It is not possible to attribute a simple profile to it, which is constant on a flux surface. ${ }^{14}$ Possibly it is due to a helical energy flow during the relaxation which at the time is asymmetric and involves several eigenfunctions.

Another example of the SVD components is shown in Fig. 9. An inward-shifted plasma with slow $(500-1000 \mathrm{~Hz})$ MHD oscillations is observed. Oscillating components (V2V4) attract much attention. They have a pronounced $m=2$ structure. A rotating structure with $m=2$ can be constructed from these three components (lower rows in Fig. 10). This is consistent with a series of simulated images assuming that the structure has a form of $\exp (-i(m \theta+n \phi)+\delta) \times \exp (-(\rho$ $\left.-\rho_{r s}\right)^{2} / \Delta \rho^{2}$ ). With parameters that fit best, $n / m=1 / 2, \rho_{r s}$ $\sim 0.4$ and $\Delta \rho \sim 0.1$, the rotating $m=2$ patterns are reproduced as shown in the upper row in Fig. 10. The mode structure $n / m=1 / 2$ is also confirmed by the magnetic probe array.

We have been able to successfully extract relevant fluctuating components using SVD, even though the fluctuation level is comparable with that of the noise. SVD is superior to fast Fourier transform in our analyses, because it automatically picks the set of eigenfunctions relevant for the process.

\section{CONCLUSION}

We have been able to obtain 2D moving pictures of MHD activities on LHD, for the case when the fluctuation amplitude is sufficiently large $(\sim 1 \%)$ and the frequency is below $2 \mathrm{kHz}$. The basic goal of the tangential viewing camera-direct visualization of fluctuations-has been achieved. This was possible even when the fluctuation amplitude was comparable to the noise level. Due to technical limitations, the framing rate, and the dynamic range of the video camera, this diagnostic works well only for a limited set of plasma parameters in the LHD. We have a plan to overcome the limitations by a newly developed video camera with a 10-bit dynamic range and a $20-\mathrm{kHz}$ framing rate, which will be installed in the next experimental campaign. Plasmas in a wider parameter space can then be investigated. Experiments on TEXTOR-94 tokamak after the end of the next experimental campaign are planned as well. A tokamak has the advantages of a simpler geometry and a more pronounced MHD activity. Furthermore, TEXTOR is presently being equipped with a helical divertor ${ }^{15}$ and then we hope to get pronounced structures on demand. 


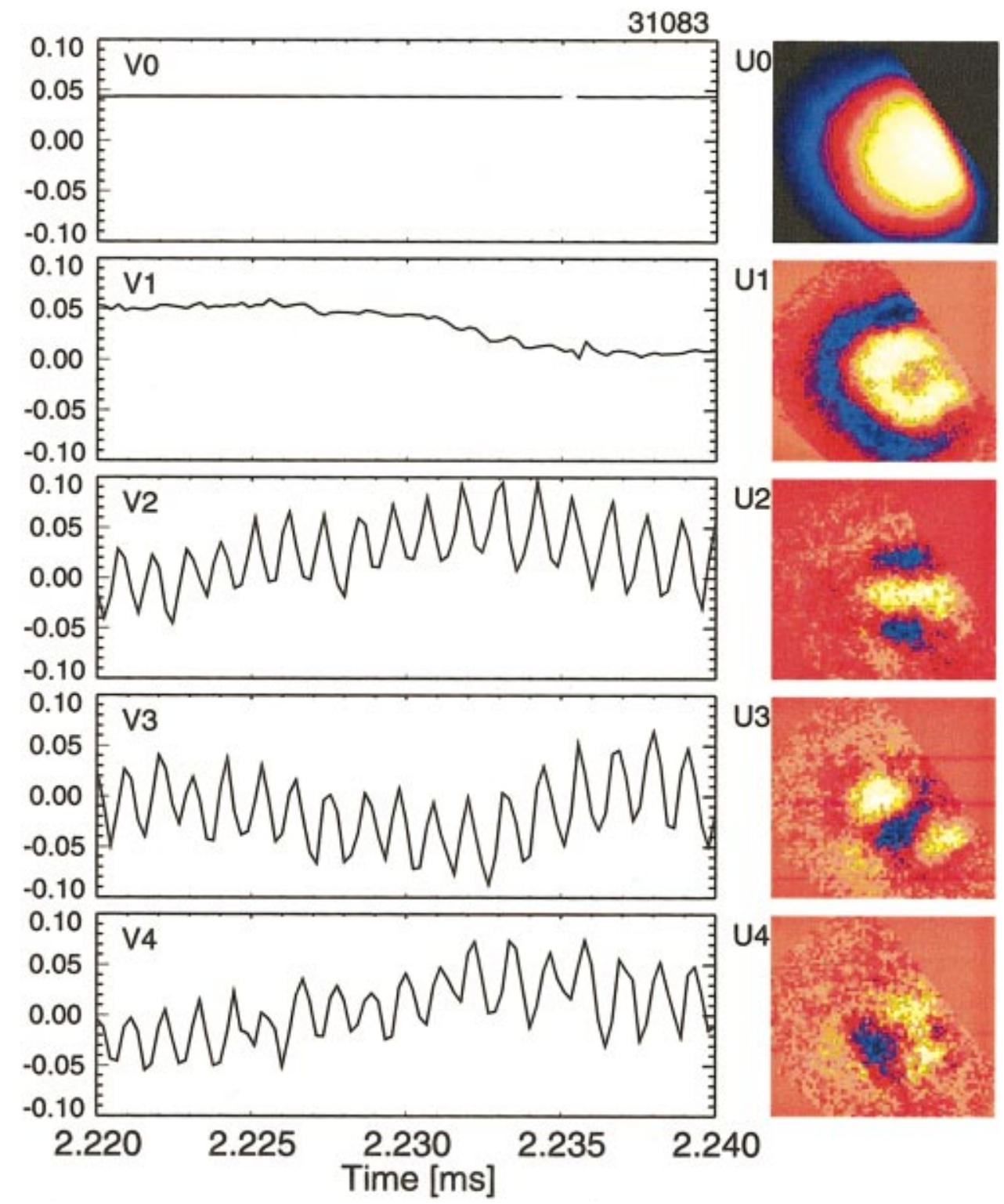

FIG. 9. (Color) Major components of the topos(U0-U4) and the chronos(V0-V4) for $R_{a x}=3.53 \mathrm{~m}$ experiments. In V2-V4, oscillating components having a $m=2$ structure (U2-U4) can be seen.

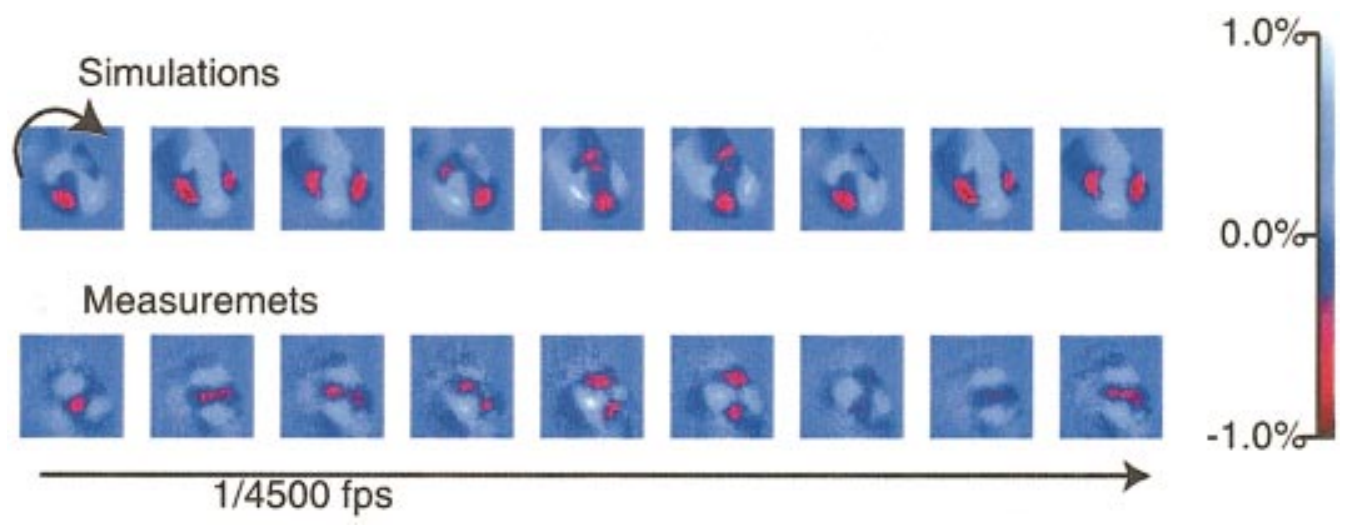

FIG. 10. (Color) Rotation of a $m=2$ perturbation extracted by the SVD method are shown in the lower row. The upper row gives simulated images by a mode with $n / m=1 / 2$. 
${ }^{1}$ S. von Goeler et al., Rev. Sci. Instrum. 61, 3055 (1990).

${ }^{2}$ D. H. J. Goodall et al., Plasma Phys. Controlled Fusion 26, 789 (1984).

${ }^{3}$ S. von Goeler et al., Rev. Sci. Instrum. 70, 602 (1999).

${ }^{4}$ G. Fuchs et al., in Controlled Fusion and Plasma Physics, Proceedings of the 26th EPS Conference, Madstricht, 1999, ECA Vol. 23J, p. 757.

${ }^{5}$ S. Sakakibara et al., Nucl. Fusion 41, 1177 (2001).

${ }^{6}$ S. Ohdachi et al., Rev. Sci. Instrum. 72, 724 (2001).

${ }^{7}$ S. Ohdachi et al., Rev. Sci. Instrum. 72, 727 (2001).

${ }^{8}$ C. Nardone, Plasma Phys. Controlled Fusion 34, 1447 (1992).

${ }^{9}$ G. Fuchs, Y. Miura, and M. Mori, Plasma Phys. Controlled Fusion 36, 307 (1994).
${ }^{10}$ S. R. Deans, The Radon Transform and Some of its Applications (Wiley, New York, 1983).

${ }^{11} \mathrm{C}$. Gorner et al., in Controlled Fusion and Plasma Physics, Proceedings of the 24th EPS Conference, Berchtesgarden, 1999, Vol. 21A, p. 1617.

${ }^{12}$ C. Fenzi et al., Rev. Sci. Instrum. 72, 989 (2001).

${ }^{13} \mathrm{~S}$. Ohdachi et al., Proceedings of 13th Stellarator Workshop, Canberra, 2002;

[http://wwwrsphysse.anu.edu.au/admin/stellarator/proceedings.html].

${ }^{14}$ Similar findings near the $t=1$ surface have been made with the soft x-ray diode array on TEXTOR, the tangential camera on TEXTOR was, up to now, too slow to observe it.

${ }^{15}$ K. Finken et al., Contrib. Plasma Phys. 40, 57 (2000). 